\title{
Managing 5FU Cardiotoxicity in Colorectal Cancer Treatment
}

\author{
Matthew Anaka, Omar Abdel-Rahman (D) \\ Department of Oncology, Cross Cancer Institute, University of Alberta, Edmonton, AB, Canada \\ Correspondence: Omar Abdel-Rahman, Department of Oncology, Cross Cancer Institute, University of Alberta, II560 University Avenue, \\ Edmonton, AB, T6G IZ2, Canada, Tel +I 780-432-8290, Fax + I 780-432-8888, Email Omar.abdelsalam@ahs.ca
}

\begin{abstract}
Fluorouracil (5FU) is the backbone chemotherapy agent in the treatment of colorectal cancer (CRC). Cardiotoxicity represents an uncommon but serious side effect of treatment with 5FU. Here, we review the current literature on 5FU-cardiotoxicity in the setting of CRC specifically, with a focus on data from the modern era of combination chemotherapy. Despite decades of study, there is little consensus on risk factors and biomarkers for 5FU-cardiotoxicity, nor how patients with CRC should be managed following a cardiotoxicity event. Given the elevated risk of recurrent cardiotoxicity on rechallenge, the use of alternative regimens that do not contain $5 \mathrm{FU}$ is a critical aspect of management. Data on the cardiotoxicity risk and efficacy of non-5FU regimens in CRC are therefore reviewed in detail.
\end{abstract}

Keywords: fluoropyrimidine, chemotherapy, adverse events, capecitabine, fluorouracil

\section{Introduction}

Fluoropyrimidines (FPD), most commonly Fluorouracil (5FU) and its derivative capecitabine, have been the backbone of chemotherapy regimens used to treat colorectal cancer (CRC) for decades. ${ }^{1,2}$ FPD-based combinations are the standard of care for palliative treatment of metastatic disease and adjuvant therapy for Stage III disease, while FPD monotherapy is employed for adjuvant treatment of high-risk stage II disease and for metastatic disease in some cases. Cardiotoxicity represents an uncommon but serious side effect of treatment with 5FU. This review will discuss the epidemiology and management of $5 \mathrm{FU}$ cardiotoxicity in $\mathrm{CRC}$ specifically, with a focus on data from the modern era of combination chemotherapy. Given the elevated risk of recurrent cardiotoxicity on rechallenge, data on the cardiotoxicity risk and efficacy of non-5FU regimens for the treatment of CRC are discussed. Areas of incomplete understanding are highlighted, with suggestions for how they might be addressed in future research.

\section{Manifestations and Pathogenesis}

Cardiotoxicity occurs most commonly during the first cycle of treatment, ${ }^{3,4}$ but can occur during later cycles as well. ${ }^{5}$ The most common cardiac manifestation of 5FU-cardiotoxicity is anginal chest pains; however, other cardiac presentations also occur. A systematic review of 30 studies found chest pain, palpitations, and dyspnea to be the most common symptoms. ${ }^{6}$ A large case series of 377 patients also identified angina (45\%), and arrhythmias (23\%) as the most common presentations, with rare presentations including acute pulmonary edema (5\%), cardiac arrest $(1.4 \%)$, and pericarditis $(1.4 \%) .{ }^{4}$ With regard to changes in cardiac investigations, $69 \%$ had evidence of ischemic electrocardiogram (ECG) changes, and $12 \%$ had abnormal cardiac enzymes. ${ }^{4}$ In patients who undergo prolonged ECG monitoring, asymptomatic ST-T changes are common, while other asymptomatic changes such as bradycardia, increased atrial and ventricular premature complexes, and QT-interval prolongation have also been reported. ${ }^{7-12}$

The pathogenesis of 5FU-induced cardiotoxicity is incompletely understood. Multiple mechanisms have been proposed, and it is likely that the impact of 5FU on the cardiovascular system is multifactorial. A systematic review has been published of 26 studies concerning the pathophysiology of $5 \mathrm{FU}$-induced cardiotoxicity. ${ }^{13}$ They grouped available evidence into several 
mechanisms, including coronary artery vasospasm, endothelial injury resulting in thrombosis, oxidative stress causing myocardial damage, and impaired oxygen transfer from red blood cells causing ischemia. The evidence for coronary vasospasm is perhaps the most well developed, with data that ranges from in vitro experiments showing vascular smooth muscle cell contraction in response to $5 \mathrm{FU},{ }^{14}$ case reports with supportive angiographic findings, ${ }^{15}$ and a study which used high-resolution ultrasound to identify brachial artery contractions after 5FU administration, which were blocked by pretreatment with nitrates. ${ }^{16}$ Endothelial damage resulting in thrombosis is likewise supported both by experimental animal data, ${ }^{17}$ as well as study using plasma samples from CRC patients being treated with FOLFOX that demonstrated reversible endothelial dysfunction causing a procoagulant state. ${ }^{18}$

Evidence of oxidative stress causing myocardial damage as a mechanism comes from pre-clinical studies, such as increased levels of superoxide anion following $5 \mathrm{FU}$ treatment of a rat heart tissue cell line, ${ }^{19}$ and decreased levels of antioxidant systems such as sodium oxide dismutase in guinea pigs treated with $5 \mathrm{FU} .{ }^{20}$ Evidence for $5 \mathrm{FU}$-induced impaired oxygen transfer from red blood cells as a potential cause of ischemia comes from the finding of decreased pO2 and increased deoxyhemoglobin in ex vivo treated erythrocytes, and increased deoxyhemoglobin in blood samples from patients treated with $5 \mathrm{FU}$ based on NMR spectroscopy analysis. ${ }^{21}$

Finally, there is evidence from rat studies that $5 \mathrm{FU}$ is metabolized by the liver into the known cardiac toxin fluoroacetate via an intermediate metabolite alpha-fluoro-beta-hydroxypropionic acid. ${ }^{22}$ A related metabolite, alphafluoro-beta-alanine (FBAL), has also been observed to be elevated in a patient who had cardiotoxicity after receiving $5 \mathrm{FU}$ as a treatment for metastatic colon cancer. ${ }^{23}$ However, another study detected fluoroacetate in the urine of $5 \mathrm{FU}$ treated patients whether or not they had cardiotoxicity. ${ }^{24}$

There is little published information concerning the mechanism underlying 5FU-induced arrhythmias. Arrhythmias may be secondary to 5FU-induced ischemia, although ECG changes independently associated with arrhythmias such as QT prolongation, increased QT dispersions, and more frequent premature complexes have also been reported in the absence of evidence of ischemia. ${ }^{7,12}$

\section{Epidemiology}

The current data describing the epidemiology of 5FU cardiotoxicity contains significant heterogeneity in terms of study setting, quality, design, and the types of malignancies and 5FU-containing regimens included. A 2013 meta-analysis of studies published from 1978 to 2012 identified rates of symptomatic 5FU cardiotoxicity ranging from $0 \%$ to $35 \%$, although the largest studies (>400 patients) reported an incidence between 1.2\% and 4.3\%. ${ }^{6}$ Most studies were observational with a high risk of bias. It is worth noting that even randomized controlled trials suffer from potential bias in reporting 5FU cardiotoxicity, given that patients with prior cardiovascular disease are often excluded from trials, and many did not specifically report on adverse cardiac events. ${ }^{25}$ In prospective monitoring studies, the incidence of ECG changes ranged between $6.0 \%$ and $44.0 \%{ }^{6}{ }^{6}$ One study identified pathologically elevated troponin levels in $57 \%$ of patients, ${ }^{26}$ however, two others reported no change. ${ }^{7,27}$

There are fewer studies which focus on CRC specifically, although these more recent analyses appear to identify a higher rate of cardiotoxicity than the studies reviewed in Polk et al. ${ }^{6}$ Three papers described pooled analyses from randomized clinic trials (RCTs). One included 3223 patients with metastatic CRC from 5 RCTs involving 1st line FPD combination chemotherapy identified cardiotoxicity in 255 patients (7.9\%). ${ }^{28}$ Another pooled 3 RCTs of capecitabine and capecitabine combinations included 1973 patients and had an incidence rate of $5.9 \% .{ }^{29}$ A pooled analysis of two trials of CAPOX for CRC included 153 patients, identifying an incidence of $6.5 \%{ }^{3}$ Several prospective observational studies limited to CRC patients have also been completed. One study of 25 patients identified no echocardiographic changes after a course of adjuvant 5FU and reported a symptomatic cardiotoxicity incidence of $8 \%{ }^{30}$ A study of 106 patients with CRC being treated adjuvant FOLFOX4 found that while most patients had a rise in NT-proBNP after administration of chemotherapy (48\% of which exceeded the assay reference range), only nine (8.5\%) had corresponding cardiac symptoms and ECG changes. ${ }^{5}$

\section{Outcomes}

Data on outcomes following 5FU-cardiotoxicity are sparse. Beyond mentions in individual case studies, there is no clear picture of how often cardiotoxicity-related concerns result in presentation to emergency departments, admission to hospital, or admission to a specialized cardiac or intensive care units. 
Studies on heart function as an outcome are mixed. A prospective echocardiographic study of 25 patients who received 5FU as adjuvant therapy for colon cancer found no changes at the end of treatment or 6 months after treatment. ${ }^{30}$ Another prospective study of 106 patients with colorectal cancer treated with adjuvant FOLFOX4 showed no changes on left ventricular ejection fraction (LVEF). ${ }^{5}$ A small prospective study found no changes in systolic or diastolic function in 12 patients treated with $5 \mathrm{FU} .^{7}$ However, these studies did not specifically address changes in cardiac function in patients who had experienced symptoms of cardiotoxicity. A prospective study of 102 patients treated with 5FU identified 19 patients who developed cardiac toxicity, 2 of whom developed reduced LVEF, which did not recover with time. ${ }^{11}$ Another study identified hypokinesia in 9 of 16 patients tested with echocardiography following the onset of cardiac symptoms, which was reversible in some but not all cases. ${ }^{24}$

Death is the outcome with the most available data. In a compilation of data from 377 patients in case reports and case series, the incidence of death in patients experiencing cardiotoxicity was $8 \%$, and $13 \%$ of patient re-exposed to $5 \mathrm{FU}$ after experiencing cardiotoxicity died. ${ }^{4}$ Another retrospective series of 5FU alone or in combination included 301 patients, 60 of which developed symptomatic cardiotoxicity, 9 of which died, for a $15 \%$ mortality rate. ${ }^{31}$ Results from prospective studies including multiple cancer types are variable, and range from a mortality rate in symptomatic patients from $0 \%$ to $28.6 \%{ }^{24,32-35}$ Two studies reported cardiotoxicity-related mortality in clinical trials of colon cancer patients. The first was a pooled analysis of two Phase III trials comparing capecitabine vs $5 \mathrm{FU} .{ }^{36}$ Of 1189 patients, 39 experienced cardiotoxicity, of which 2 died (mortality rate 5.1\%). In the second pooled analysis including 153 patients from two trials investigating CAPOX for the treatment of metastatic colorectal cancer, 10 patients experienced cardiotoxicity, of which 2 died (mortality rate $20 \%)^{3}$

\section{Risk Factors}

Attempts to identify patient-level risk factors predictive of 5FU-cardiotoxicity have not resulted in any reliable measures. Pre-existing cardiac risk factors or a history of cardiovascular disease is not consistently associated with higher rates of 5FU-cardiotoxicity. There are retrospective series which identified association between cardiac risk factors, cardiac disease and 5FU cardiotoxicity, ${ }^{34,37}$ although other retrospective and prospective studies report no significant associations. ${ }^{4,35,38}$ Increasing age is not clearly associated with increased risk. ${ }^{28,34,37,39}$ In fact, one large single centre retrospective series of 4019 patients treated with 5FU actually identified significantly increased incidence in younger patients, who were also significantly less likely to have cardiac risk factors, and less likely to be on cardioprotective medications. ${ }^{40}$ In colorectal cancer specifically no association was seen between cardiac disease or risk factors and the level of NT-proBNP rise after $5 \mathrm{FU},{ }^{5}$ or with cardiac risk factors or cardiac disease in two separate pooled clinical trial analyses. ${ }^{3,28}$

In regard to the risks associated with specific regimens, longer infusions of 5FU appear to be associated with a higher incidence of cardiotoxicity than shorter infusions $\left(6.3\right.$ vs $2.2 \%$ in one study $\left.{ }^{33}\right)$. Older studies of bolus $5 \mathrm{FU}$ regimens reported rates between $1.6 \%$ and $3 \% .{ }^{37,41}$ Dose does not seem to be related to cardiotoxicity. ${ }^{34}$ Capecitabine appears to be associated with a similar incidence of cardiotoxicity to $5 \mathrm{FU}$, with reported incidence ranging from 3\% to $5.5 \% .{ }^{29,33,36}$

Combination of 5FU with other chemotherapy agents represents another potential risk factor. Individual studies involving multiple cancer types have identified an increased risk of cardiotoxicity for 5FU in combination with etoposide $^{34}$ and cisplatin. ${ }^{31}$ In CRC specifically, FPD combination regimens appear to be associated with increased cardiotoxicity. An incidence of $6.5 \%$ was identified in patients treated with CAPOX across two trials. ${ }^{3}$ A pooled analysis of 5 CRC trials included patients treated with FOLFOX or FOLFIRI in combination with either bevacizumab and panitumumab. ${ }^{28}$ On multivariate regression analysis, only bevacizumab and panitumumab regimens were predictive of cardiotoxicity. Bevacizumab-containing regimens were significantly more likely to be associated with any cardiac toxicity $(9.8 \%$ vs $6.1 \%)$, specifically ischemic events $(2.9 \%$ vs $1 \%)$. Panitumumab-containing regimens were significantly more likely to be associated with any cardiac toxicity (11.5\% vs $6.6 \%)$, and specifically arrhythmias ( $7.5 \%$ vs $3.7 \%){ }^{28}$ Another pooled analysis of 3 clinical trials of capecitabine and capecitabine combination regimens in CRC found that CAPOX + bevacizumab was associated with the highest rate of cardiotoxicity ( $12 \%$ vs $4 \%$ for capecitabine monotherapy), with the caveat the CAPOX + bevacizumab + cetuximab had a cardiotoxicity incidence of $7 \% .{ }^{29}$ It is not clear whether the increased incidence of cardiotoxicity with the addition of biologics is synergistic or additive, given that 
these agents are associated independently with cardiac adverse events. Cetuximab and panitumumab have previously been associated with increased risk of new-onset heart failure. ${ }^{42}$ Panitumumab is also known to induce electrolyte disturbances, in particular hypomagnesemia, which may contribute to the increased incidence of arrhythmias. ${ }^{43}$ Bevacizumab, along with other anti-VEGF agents, has likewise been independently associated with heart failure, myocardial ischemia, and other arterial thromboembolic events in large metanalyses. ${ }^{4,45}$

Data on any potential relationship between dihydropyrimidine dehydrogenase (DPD) polymorphisms and 5FU cardiotoxicity are sparse. A retrospective study of 59 patients with unanticipated toxicity from 5FU found that 19 had DPD deficiency, only 1 of which had cardiotoxicity. ${ }^{46}$

\section{Primary Prevention}

Efforts towards primary prevention of 5FU-cardiotoxicity are hampered by the lack of consistent data on risk factors for its development. Nevertheless, this issue must be considered by treating clinicians both in routine practice and by those developing clinic trial protocols. A review of clinical trials that involved 5FU or capecitabine from ECOG-ACRIN Cancer Research Group found that pre-existing cardiovascular disease was an exclusion criterion in 13/16 trials. ${ }^{25}$ Participants with recent acute coronary syndrome (ACS; unstable angina or myocardial infarction) were excluded in 11 studies, with the definition of "recent" ranging from 3 to 12 months prior to enrollment. Guidelines, such as those from the Canadian Cardiovascular Society (CCS) on the evaluation and management of cardiovascular complications of cancer therapy, make no specific recommendations about deferring treatment with 5FU after ACS. ${ }^{47}$

There are little data on the ability of pre-treatment investigation to predict patients who may develop cardiotoxicity. A small prospective study which enrolled 52 patients treated with capecitabine found that an abnormal echocardiogram at baseline was associated with the development of cardiotoxicity. ${ }^{48}$ The significantly higher increase in NT-proBNP following 5FU treatment in patients who went on to develop cardiac toxicity suggests a potential for this test to become a predictive biomarker, ${ }^{5}$ although it has not yet been evaluated for this use. Data on the prophylactic use of cardioprotective medications are all in the setting of secondary prevention and are described below. In the absence of robust data, attempts at primary prevention remain empiric, and largely consist of a clinical judgement as to which patients may have a relative contraindication to receiving $5 \mathrm{FU}$.

\section{Secondary Prevention}

Rechallenge in patients with 5FU-induced cardiotoxicity appears to carry a significant risk of recurrent cardiac events, with one case series reporting the incidence of recurrence to be $90 \%{ }^{49}$ Severe outcomes may also be more common. A review of 377 cases found that $13 \%$ of patients who were rechallenged following initial 5FU-related cardiac events died from recurrent toxicity, vs an $8 \%$ mortality rate for initial events. ${ }^{4}$ Rechallenge must therefore be carefully considered, and a variety of efforts have been made to reduce the incidence of recurrent cardiotoxicity upon rechallenge. One case series of 5 patients reports of secondary prevention of capecitabine-associated chest pain with diltiazem, ${ }^{50}$ but a study of primary prevention using this agent in 58 patients being treated with cisplatin and $5 \mathrm{FU}$ reported a $12 \%$ incidence of cardiotoxicity, similar to the $13 \%$ incidence in their chosen historical controls. ${ }^{51}$ There are case reports of using nitrates in the secondary prevention setting with mixed outcomes. ${ }^{52,53}$ CCS guidelines do not explicitly recommend the use of prophylactic vasodilators. ${ }^{47}$

Dose reduction does not appear to be effective in preventing recurrent toxicity, ${ }^{4}$ and in keeping with data that suggests there is no dose-relationship between 5FU and cardiotoxicity. ${ }^{34}$ Infusional $5 \mathrm{FU}$ is associated with more cardiotoxicity than bolus $5 \mathrm{FU}$, and there is limited evidence that switching from infusional $5 \mathrm{FU}$ to a bolus $5 \mathrm{FU}$ regimen can prevent recurrent toxicity. This includes a single centre case series that demonstrated no recurrent cardiotoxicity in six patients who were switched from FOLFOX to FLOX, where 5FU is given as a weekly bolus. ${ }^{54}$ Given the paucity of data available on rechallenge, decisions on who to rechallenge are empiric. Factors to consider include the severity of the initial event, the intent of treatment (curative vs palliative), and the availability and efficacy of alternative agents with a lower risk of cardiotoxicity. 


\section{Cardiotoxicity of Alternative Agents}

Given the paucity of reliable data on risk factors, biomarkers, and prophylaxis during rechallenge, the primary means of primary and secondary prevention of 5FU-cardiotoxicity for patients with CRC involves the use of chemotherapy regimens that do not include 5FU. This is especially challenging in the management of patients with CRC, given that $5 \mathrm{FU}$ is the backbone of the chemotherapy regimens used in all disease stages. Understanding the risk-benefit profile of any decision to deviate from well established standard regimens requires an understanding of the associated cardiotoxicity risk of the involved chemotherapy agents, and the efficacy of alternative agents relative to $5 \mathrm{FU}$ in the treatment of CRC.

There are case reports of successful treatment with capecitabine following 5FU-related cardiotoxicity, ${ }^{55,56}$ but more robust evidence is lacking. Given what appears to be an equivalent risk of cardiotoxicity with capecitabine as an initial therapy described above, in the absence of more robust data supporting safety switching from 5FU to capecitabine, it cannot be recommended if there are any viable alternatives. Switching to either an alternative FPD with a lower risk of cardiotoxicity, or a non-fluoropyrimidine containing regimen, represent safer options from a cardiotoxicity perspective.

UFT is a combination of fluorouracil prodrug ftorafur and uracil, which serves to inhibit fluorouracil degradation. Reported cardiotoxicity across multiple studies is less than $1 \% .{ }^{57-60}$ However there are no prospective or large retrospective series supporting its use following $5 \mathrm{FU}$ cardiotoxicity, and a death secondary to congestive heart failure has been observed following this approach. ${ }^{38}$ Availability can also be an issue; UFT is not approved for use in North America.

$\mathrm{S} 1$ is another oral fluoropyrimidine that contains ftorafur, in combination gimeracil and oteracil. Gimeracil is a DPD inhibitor, which prevents the breakdown of fluorouracil into FBAL, ${ }^{61}$ which as described above is implicated in $5 \mathrm{FU}$ induced cardiotoxicity. Remarkably, there are no reports of cardiotoxicity following treatment with S1. ${ }^{62-64}$ There are case reports of successful use of S1 after 5FU-induced cardiotoxicity, ${ }^{23}$ but no larger case series. Availability is also limited to some Asian and European countries.

Trifluridine/tipiracil (TAS-102) contains an oral fluoropyrimidine (trifluridine) and a thymidine phosphorylase inhibitor (tipiracil). Among the 534 patients treated with trifluridine/tipiracil in the phase III trial supporting its use in metastatic colorectal cancer, only 2 patients developed cardiac ischemia $(0.37 \%)$, vs 1 of 266 patients treated with placebo $(0.38 \%) .{ }^{65}$ An abstract describing a systematic review and meta-analysis of 1877 patients treated in 4 trials has been published, and found no increase in cardiac events relative to placebo. ${ }^{66}$ There are no available reports of treatment with Trifluridine/tipiracil after 5FU-induced cardiotoxicity.

Raltitrexed is an antimetabolite chemotherapy agent that functions through inhibition of thymidylate synthase. A systematic review of cardiotoxicity in patients treated with raltitrexed included four pivotal trials in CRC and pancreatic cancer, with no reported cardiotoxicity. ${ }^{67}$ In the same study, a retrospective review of raltitrexed included 111 patients where raltitrexed was used first line due to elevated cardiovascular risk, or second line after FPD-related cardiotoxicity. It found a cardiovascular event rate of $4.5 \%$ (5/111 patients) in a presumably high-risk population. ${ }^{67}$ Four of those patients died, apparently of events related to disease progression, although more details were lacking. In contrast, another retrospective review of raltitrexed use following FPD-cardiotoxicity included 42 patients and reported no cardiac events. ${ }^{68}$ Regardless of the potential reduction in cardiotoxicity, caution is warranted with raltitrexed given that treatment-related mortality was as high as $6 \%$ in some clinical trials. ${ }^{69}$

\section{Efficacy of Alternative Regimens in CRC}

While switching to a non-FPD containing regimen or an alternative FPD with a lower risk of cardiotoxicity likely represent safer options from a cardiotoxicity perspective, a full picture of the risk/benefit profile of this strategy requires an understanding of their relative efficacy in the treatment of CRC patients.

Given the curative intent of treatment, cardiotoxicity and resulting modifications to adjuvant regimens for resected CRC are of particular concern. Results of Phase III studies of alternative agents are shown in Table 1. Two large, randomized studies have shown equivalent results with UFT and leucovorin (LV) in comparison to bolus 5FU/LV in the adjuvant treatment of CRC. ${ }^{70,71}$ Data on adjuvant S1 in CRC are mixed. Adjuvant S1 was found to be non-inferior to UFT in the treatment of Stage III CRC patients in a randomized phase III study. ${ }^{72}$ However, S1 was not found to be noninferior to capecitabine in a randomised Phase 3 trial of adjuvant therapy for stage III CRC, with capecitabine showing 
Table I Summary of Phase III Studies of FPD Alternatives for Adjuvant Treatment of CRC

\begin{tabular}{|l|l|l|l|l|l|l|}
\hline Regimen & Comparator & Primary Outcome & OS at 5 Years & DFS at 5 Years & DFS at 3 Years & Reference \\
\hline UFT & FFU/LV bolus & $\begin{array}{l}\text { OS. } \\
\text { HR I.0I4; P }=0.90 .\end{array}$ & 78.5 & 67.0 & - & {$[70]$} \\
\hline UFT & 5FU/LV bolus & $\begin{array}{l}\text { DFS. } \\
\text { HR I.016; non-inferiority P }=0.0236 .\end{array}$ & 82.4 & 73.6 & - & {$[7 /]$} \\
\hline SI & UFT & $\begin{array}{l}\text { DFS at 3 years. } \\
\text { HR 0.85; non-inferiority P }<0.00 I .\end{array}$ & - & - & 72.5 & {$[72]$} \\
\hline SI & Capecitabine & $\begin{array}{l}\text { DFS at 3 years. } \\
\text { HR I.23, non-inferiority P }=0.46 .\end{array}$ & - & - & 77.9 & {$[73]$} \\
\hline Raltitrexed & Bolus 5FU/LV & $\begin{array}{l}\text { Relapse-free survival. } \\
\text { HR I.16. }\end{array}$ & $61.9 \%$ & - & - & {$[83]$} \\
\hline
\end{tabular}

superior disease-free survival at an interim analysis. ${ }^{73}$ There are no Phase III studies incorporating oxaliplatin in combination with an alternative agent to replace 5FU. As rates of cardiotoxicity are apparently higher with infusional $5 \mathrm{FU}$, switching to a bolus 5FU bolus with evidence in the adjuvant setting is another option. For stage III patients for whom an oxaliplatin-containing adjuvant regimen is indicated, the use of FLOX represents a bolus-5FU alternative. Adjuvant FLOX appears to have similar efficacy to FOLFOX, albeit with a different toxicity profile. ${ }^{74}$ There are reports of the use of FLOX following cardiotoxicity with adjuvant infusion 5FU, with no recurrent cardiac events. ${ }^{75}$ For patient with Stage II CRC, the Roswell Park regimen is an established bolus 5FU-monotherapy alternative. ${ }^{76}$

There are more options available in the metastatic setting, with relevant Phase III studies summarized in Table 2 (firstline studies) and Table 3 (second line and later studies). UFT appeared comparable to bolus 5FU in the metastatic setting. ${ }^{77}$ UFT combinations with oxaliplatin and irinotecan likewise appear comparable to FOLFOX and FOLFIRI in the treatment of mCRC, although data is limited to Phase II studies. ${ }^{78}$

Data supporting single agent S1 in the treatment of metastatic CRC come from two single-arm studies showing evidence of objective responses. ${ }^{79,80} \mathrm{~S} 1$ and oxaliplatin were found to be non-inferior to capecitabine/oxaliplatin in a phase III study of patients with advanced CRC. ${ }^{81} \mathrm{~S} 1$ and irinotecan was likewise found to be non-inferior to FOLFIRI in a randomized phase II/III study of $\mathrm{mCRC}$ in the second line. ${ }^{82}$

A phase III trial comparing raltitrexed to bolus $5 \mathrm{FU}$ in the adjuvant treatment of stage III CRC failed to show noninferiority after early termination, with raltitrexed associated with a lower relapse free survival and high toxicity in the setting significant protocol deviations related to adjustment for creatinine clearance. ${ }^{83}$ Two phase III studies comparing raltitrexed to $5 \mathrm{FU}$ in advanced $\mathrm{CRC}$ patients showed similar response rates, although with one showing a significantly shorter PFS for raltitrexed. ${ }^{84,85}$ Another phase III study in the metastatic setting found raltitrexed to have a similar overall survival to the de Gramont regimen, but significantly worse quality of life and an increase in treatment-related deaths. ${ }^{86}$ Raltitrexed has been studied in combination with both oxaliplatin and irinotecan. In a Phase II study described in an abstract only, TOMOX was compared to FOLFOX in the first-line treatment of metastatic CRC. There were no significant differences in response rate, time to progression, or overall survival. ${ }^{87}$ Two single arm trials of second-line treatment with TOMOX after progression on 5-FU likewise showed evidence of activity. ${ }^{88,89}$ In addition, a single centre retrospective case series describing use of TOMOX after discontinuation of 5FU-based treatment due to severe toxicity in 44 patients found a three year relapse free of $70.8 \%$ and overall survival of $83.6 \% .{ }^{90}$ The combination of raltitrexed and irinotecan has also been investigated in the metastatic setting. In a Phase II trial comparing raltitrexed and irinotecan with TOMOX, there were no significant differences in efficacy measures between the regimens, with response rates of $46 \%$ and $34 \%$, respectively. ${ }^{91}$ Further data on raltitrexed + irinotecan comes from two single arm Phase II studies in the firstline setting, with comparable results. ${ }^{92,93}$ Raltitrexed + irinotecan was also shown to be active after progression on 5FU, with a response rate of $15.4 \%$ in a single arm study. ${ }^{94}$ 
Table 2 Summary of Phase III Studies of FPD Alternatives for First-Line Treatment of Metastatic CRC

\begin{tabular}{|c|c|c|c|c|c|c|}
\hline Regimen & Comparator & Primary Outcome & $\begin{array}{l}\text { ORR } \\
\text { (\%) }\end{array}$ & $\begin{array}{l}\text { PFS } \\
\text { (Months) }\end{array}$ & $\begin{array}{l}\text { Overall Survival } \\
\text { (Months) }\end{array}$ & Reference \\
\hline UFT & Bolus 5FU/LV & $\begin{array}{l}\text { OS. } \\
\text { HR 0.964; } P=0.630 \text {. }\end{array}$ & 11.7 & 3.5 & 12.4 & [77] \\
\hline UFT & Bolus 5FU/LV & $\begin{array}{l}\text { PFS. } \\
\text { Logrank } P=0.591 .\end{array}$ & 10.5 & 3.4 & 12.2 & {$[114]$} \\
\hline Raltitrexed & Bolus 5FU/LV & $\begin{array}{l}\text { PFS. } \\
\text { HR I.I5; P=0.197. }\end{array}$ & 19 & 3.9 & 10.9 & [84] \\
\hline Raltitrexed & Bolus 5FU/LV & $\begin{array}{l}\text { PFS. } \\
\text { HR I.08; P=0.44. }\end{array}$ & 19.3 & 4.7 & 10.3 & [85] \\
\hline Raltitrexed & Infusional 5FU/LV & $\begin{array}{l}\text { OS. } \\
\text { HR 0.99; P=0.94. }\end{array}$ & 18 & 4.8 & 8.7 & [86] \\
\hline Irinotecan & $\begin{array}{l}5 \mathrm{FU} / \mathrm{LV} \text { and } 5 \mathrm{FU} / \mathrm{LV} / \\
\text { irinotecan }\end{array}$ & $\begin{array}{l}\text { PFS. } \\
\text { Statistics not shown for } \\
\text { irinotecan vs 5FU/LV. }\end{array}$ & 18 & 4.3 & 12 & [99] \\
\hline $\begin{array}{l}\text { Irinotecan + } \\
\text { oxaliplatin (IROX) }\end{array}$ & Irinotecan + 5FU/LV & $\begin{array}{l}\text { PFS. } \\
\text { HR I. } 14 ; P=0.178 \text {. }\end{array}$ & 41 & 7.2 & 19 & {$[104]$} \\
\hline SI and oxaliplatin & $\begin{array}{l}\text { Capecitabine + } \\
\text { oxaliplatin }\end{array}$ & $\begin{array}{l}\text { PFS. } \\
\text { HR } 0.809 \text {, non-inferiority } P= \\
0.087 .\end{array}$ & 48.9 & 7.1 & 20.9 & [8I] \\
\hline
\end{tabular}

Table 3 Summary of Phase III Studies of FPD Alternatives in Second Line or Later Treatment of Metastatic CRC

\begin{tabular}{|c|c|c|c|c|c|c|}
\hline Regimen & Comparator & Primary Outcome & $\begin{array}{l}\text { ORR } \\
\text { (\%) }\end{array}$ & $\begin{array}{l}\text { PFS } \\
\text { (Months) }\end{array}$ & $\begin{array}{l}\text { Overall Survival } \\
\text { (Months) }\end{array}$ & Reference \\
\hline Irinotecan (after 5FU) & BSC & $\begin{array}{l}\text { OS. } \\
\text { Logrank } \mathrm{P}=0.000 \mathrm{I} .\end{array}$ & - & - & 9.2 & {$[100,104]$} \\
\hline Irinotecan (after $5 \mathrm{FU}$ ) & Infusional 5FU & OS. Logrank $\mathrm{P}=0.035$. & - & 4.2 & 10.8 & {$[101]$} \\
\hline $\begin{array}{l}\text { Irinotecan }+ \text { cetuximab (after } \\
\text { irinotecan-based regimen) }\end{array}$ & Cetuximab & $\begin{array}{l}\text { ORR. } \\
P=0.007\end{array}$ & 22.9 & 4.1 & 8.6 & {$[102]$} \\
\hline $\begin{array}{l}\text { Irinotecan + cetixumab (after } \\
\text { FOLFOX) }\end{array}$ & Irinotecan & $\begin{array}{l}\text { OS. } \\
\text { HR 0.975; P=0.7I. }\end{array}$ & 16.4 & 4.0 & 10.7 & [103] \\
\hline IROX (after 5FU) & Irinotecan & $\begin{array}{l}\text { OS. } \\
\text { HR } 0.78 ; P=0.0072 \text {. }\end{array}$ & 22 & 5.3 & 13.4 & {$[105]$} \\
\hline $\begin{array}{l}\text { SI + irinotecan (after } 5 \mathrm{FU} \pm \\
\text { oxaliplatin) }\end{array}$ & FOLFIRI & $\begin{array}{l}\text { PFS. } \\
\text { HR I.077, non-inferiority } \\
P=0.039 .\end{array}$ & 18.8 & 5.8 & 19.5 & [82] \\
\hline $\begin{array}{l}\text { TAS-I02 (3rd line, after 5FU, } \\
\text { oxaliplatin, irinotecan) }\end{array}$ & Placebo & $\begin{array}{l}\text { OS. } \\
\text { HR } 0.68 \mathrm{P}<0.001 \text {. }\end{array}$ & 1.6 & 2.0 & 7.1 & [65] \\
\hline
\end{tabular}

Trifluridine/tipiracil was initially evaluated in patients with metastatic CRC who had progressed on standard chemotherapy regimens include, making it difficult to compare the efficacy of trifluridine/tipiracil and traditional FPDs. Trifluridine/tipiracil has a demonstrated overall survival benefit vs placebo in patients who have progression on 5FU, oxaliplatin, and irinotecan. ${ }^{65}$ A randomized Phase II trial in metastatic CRC patient not eligible for standard first- 
line 5FU-doublet chemotherapy compared trifluridine/tipiracil plus bevacizumab and capecitabine plus bevacizumab, and found comparable efficacy. ${ }^{95,96}$ Randomized Phase III studies in the first and second line are ongoing. ${ }^{97,98}$

Irinotecan is active as a single agent in metastatic CRC. In the first-line setting, outcomes are similar to 5FU/LV, although inferior to a 5FU/LV + irinotecan regimen. ${ }^{99}$ In two second-line Phase III studies, irinotecan has activity after progression on 5FU. ${ }^{100,101}$ Two Phase III studies also demonstrated evidence of activity for second-line irinotecan + cetuximab after progression on either FOLFOX or an irinotecan-5FU regimen, ${ }^{102,103}$ although overall survival results are comparable to irinotecan as a single agent. Irinotecan + oxaliplatin (IROX) is another option with Phase III data. First-line IROX had comparable results to a first-line regimen of infusional 5FU, leucovorin, and irinotecan. ${ }^{104}$ In the second line after progression on 5FU, IROX showed significant improvement of OS relative to single agent irinotecan. ${ }^{105}$

\section{Treatment of Cardiotoxicity}

There are little data on which to guide the acute management or work-up of 5FU-related cardiotoxicity, and suggested strategies are therefore extrapolated from other settings, and primarily reflect expert opinion. Unfortunately, many cardiooncology guidelines from major organizations contain little to no information on 5FU-related cardiotoxicity. ${ }^{106-108}$ CCS guidelines present the most comprehensive approach. ${ }^{47}$ If symptoms of ischemia occur during a 5FU-infusion, the infusion should be stopped immediately. Symptoms should be treated with nitroglycerin, as well as opioids if needed. The initial workup should include an ECG and troponin levels, and patient should be placed on cardiac monitors. If the troponin is elevated, patients should be managed using established algorithms for ACS. If there is no evidence for ACS, elective work-up for underlying coronary artery disease can be considered. Given the lack of specific biomarkers, the diagnosis of 5-FU cardiotoxicity requires a plausible temporal relationship between drug and symptoms. If a diagnosis of 5FU-cardiotoxicity is apparent, rechallenge should only be considered if no alternative treatments are available. Rechallenge should be performed in a monitored setting, include dose reduction or transition to a bolus regimen, consideration made of prophylaxis with nitrates or calcium channel blockers despite the mixed evidence. ${ }^{47}$

Other individual researchers have proposed algorithms for work-up. One suggests work-up stratified by the patient's underlying risk for coronary artery disease (CAD). ${ }^{109}$ Lower risk patients would undergo non-invasive testing such as CT coronary angiogram, while high-risk patient would undergo coronary angiogram to attempt to identify a contributing coronary lesion. No finding of significant CAD supports a diagnosis of 5FU-cardiotoxicity, and alternative treatment strategies would be pursued. For patients with significant CAD that might contribute to symptoms, revascularization may be followed by cautious rechallenge with close monitoring and prophylaxis with vasodilators. ${ }^{109}$

Uridine triacetate was developed as an antidote for fluoropyrimidine overdose. Uridine triacetate is a prodrug, which is metabolically converted into uridine and competes with incorporation of 5FU into RNA, attenuating toxicity. ${ }^{110}$ A summary of two single arm, open label trials using uridine triacetate to treat $5 \mathrm{FU}$ overdose in 135 patients, primarily due to dose miscalculations and pump errors, has been published. ${ }^{111}$ They found that the survival rate in patients treated with uridine triacetate was $96 \%$, vs $16 \%$ for a set of historical controls. This included two patients who presented with symptoms of cardiac toxicity and experienced rapid reversal of symptoms. There is further evidence for efficacy in the setting of cardiotoxicity from case reports. ${ }^{112}$ It was approved by the FDA for treatment of severe FPD toxicity in $2015 .{ }^{113}$ Further studies will be needed to determine whether uridine triacetate can be incorporated routinely into the management of patients' 5FU-cardiotoxicity.

\section{Summary}

Cardiotoxicity is a well-known toxicity of 5FU. However, despite many years of study of this long-recognized problem, we still do not have a clear picture of risk factors for its development, predictive biomarkers, or a clear approach to primary or secondary prevention. This situation is especially problematic in the setting of CRC, where 5FU and capecitabine are key components of first-line treatment options. The lack of large prospective studies comparing alternative regimens to modern 5FU-combination chemotherapy is therefore another major obstacle in the management of 5FU-cardiotoxicity in CRC. Unfortunately, the risk of cardiotoxicity appears higher with the combination regimens used in $\mathrm{CRC}$, in particular those that incorporate bevacizumab and panitumumab. 5FU-cardiotoxicity therefore remains 
an issue that should be prioritized for further study. We believe that rigorous collection of information on cardiotoxicity and patient-level cardiac risk factors should be a part of every CRC clinical trial that incorporates FPDs, even when they are not the experimental agent. Prospective studies to evaluate predictive biomarkers, including modern cardiac imaging tests, are needed. Strategies such as prophylaxis with cardioprotective medications during rechallenge also require more extensive validation. Data on alternative adjuvant regimens for Stage III CRC are lacking. In the metastatic setting combinations of S1 and oxaliplatin or irinotecan appear comparable to their 5FU counterparts based on data from RCTs, and the IROX regimen represents a means to expose patients to two highly active agents. However, availability of S1, and a lack of data on combining any of these regiments with biologics, gives an incomplete picture of their ability to replace treatments including 5FU. Management of 5FU-cardiotoxicity in CRC therefore remains an open problem.

\section{Abbreviations}

5FU, fluorouracil; ACS, acute coronary syndrome; CAD, coronary artery disease; CCS, Canadian Cardiovascular Society; CRC, colorectal cancer; DPD, dihydropyrimidine dehydrogenase; ECG, electrocardiogram; FBAL, alphafluoro-beta-alanine; FPD, fluoropyrimidine; LV, leucovorin; LVEF, left ventricular ejection fraction; RCT, randomized control trial.

\section{Disclosure}

MA reports no conflicts of interest related to the topic of this work.

OAR reports advisory board with Ipsen, Lilly, Roche, Bayer, and Eisai and no other conflicts of interest for this work.

\section{References}

1. Nordic Gastrointestinal Tumor Adjuvant Therapy Group. Expectancy or primary chemotherapy in patients with advanced asymptomatic colorectal cancer: a randomized trial. J Clin Oncol. 1992;10(6):904-911. doi:10.1200/JCO.1992.10.6.904

2. Scheithauer W, Rosen H, Kornek GV, Sebesta C, Depisch D. Randomised comparison of combination chemotherapy plus supportive care with supportive care alone in patients with metastatic colorectal cancer. BMJ. 1993;306(6880):752-755. doi:10.1136/bmj.306.6880.752

3. $\mathrm{Ng} \mathrm{M}$, Cunningham D, Norman AR. The frequency and pattern of cardiotoxicity observed with capecitabine used in conjunction with oxaliplatin in patients treated for advanced colorectal cancer (CRC). Eur J Cancer. 2005;41(11):1542-1546.

4. Saif MW, Shah MM, Shah AR. Fluoropyrimidine-associated cardiotoxicity: revisited. Expert Opin Drug Saf. 2009;8(2):191-202. doi:10.1517/ 14740330902733961

5. Jensen SA, Hasbak P, Mortensen J, Sørensen JB. Fluorouracil induces myocardial ischemia with increases of plasma brain natriuretic peptide and lactic acid but without dysfunction of left ventricle. J Clin Oncol. 2010;28(36):5280-5286. doi:10.1200/JCO.2009.27.3953

6. Polk A, Vaage-Nilsen M, Vistisen K, Nielsen DL. Cardiotoxicity in cancer patients treated with 5-fluorouracil or capecitabine: a systematic review of incidence, manifestations and predisposing factors. Cancer Treat Rev. 2013;39(8):974-984. doi:10.1016/j.ctrv.2013.03.005

7. Oztop I, Gencer M, Okan T, et al. Evaluation of cardiotoxicity of a combined bolus plus infusional 5-fluorouracil/folinic acid treatment by echocardiography, plasma troponin I level, QT interval and dispersion in patients with gastrointestinal system cancers. Jpn J Clin Oncol. 2004;34(5):262-268. doi:10.1093/jjco/hyh047

8. Rezkalla S, Kloner RA, Ensley J, et al. Continuous ambulatory ECG monitoring during fluorouracil therapy: a prospective study. J Clin Oncol. 1989;7(4):509-514. doi:10.1200/JCO.1989.7.4.509

9. Stewart T, Pavlakis N, Ward M. Cardiotoxicity with 5-fluorouracil and capecitabine: more than just vasospastic angina. Intern Med J. 2010;40 (4):303-307. doi:10.1111/j.1445-5994.2009.02144.x

10. Talapatra K, Rajesh I, Rajesh B, Selvamani B, Subhashini J. Transient asymptomatic bradycardia in patients on infusional 5-fluorouracil. $J$ Cancer Res Ther. 2007;3(3):169-171. doi:10.4103/0973-1482.37412

11. Wacker A, Lersch C, Scherpinski U, Reindl L, Seyfarth M. High incidence of angina pectoris in patients treated with 5-fluorouracil. Oncology. 2003;65(2):108-112. doi:10.1159/000072334

12. Yilmaz U, Oztop I, Ciloglu A, et al. 5-fluorouracil increases the number and complexity of premature complexes in the heart: a prospective study using ambulatory ECG monitoring. Int J Clin Pract. 2007;61(5):795-801. doi:10.1111/j.1742-1241.2007.01323.x

13. Polk A, Vistisen K, Vaage-Nilsen M, Nielsen DL. A systematic review of the pathophysiology of 5-fluorouracil-induced cardiotoxicity. $B M C$ Pharmacol Toxicol. 2014;15:47. doi:10.1186/2050-6511-15-47

14. Mosseri M, Fingert HJ, Varticovski L, Chokshi S, Isner JM. In vitro evidence that myocardial ischemia resulting from 5-fluorouracil chemotherapy is due to protein kinase C-mediated vasoconstriction of vascular smooth muscle. Cancer Res. 1993;53(13):3028-3033.

15. Kim S-M, Kwak C-H, Lee B, et al. A case of severe coronary spasm associated with 5-fluorouracil chemotherapy. Korean J Intern Med. 2012;27(3):342-345. doi:10.3904/kjim.2012.27.3.342

16. Südhoff T, Enderle M-D, Pahlke M, et al. 5-Fluorouracil induces arterial vasocontractions. Ann Oncol. 2004;15(4):661-664. doi:10.1093/ annonc/mdh 150

17. Cwikiel M, Eskilsson J, Wieslander JB, Stjernquist U, Albertsson M. The appearance of endothelium in small arteries after treatment with 5-fluorouracil. An electron microscopic study of late effects in rabbits. Scanning Microsc. 1996;10(3):805-818; discussion 819.

18. Jensen SA, Sørensen JB. 5-fluorouracil-based therapy induces endovascular injury having potential significance to development of clinically overt cardiotoxicity. Cancer Chemother Pharmacol. 2012;69(1):57-64. doi:10.1007/s00280-011-1669-x 
19. Lamberti M, Porto S, Marra M, et al. 5-fluorouracil induces apoptosis in rat cardiocytes through intracellular oxidative stress. J Exp Clin Cancer Res. 2012;31:60. doi:10.1186/1756-9966-31-60

20. Durak I, Karaayvaz M, Kavutcu M, et al. Reduced antioxidant defense capacity in myocardial tissue from Guinea pigs treated with 5-fluorouracil. J Toxicol Environ Health A. 2000;59(7):585-589. doi:10.1080/009841000156709

21. Spasojević I, Jelić S, Zakrzewska J, Bacić G. Decreased oxygen transfer capacity of erythrocytes as a cause of 5-fluorouracil related ischemia. Mol. 2008;14(1):53-67. doi:10.3390/molecules14010053

22. Arellano M, Malet-Martino M, Martino R, Gires P. The anti-cancer drug 5-fluorouracil is metabolized by the isolated perfused rat liver and in rats into highly toxic fluoroacetate. Br J Cancer. 1998;77(1):79-86. doi:10.1038/bjc.1998.12

23. Muneoka K, Shirai Y, Yokoyama N, Wakai T, Hatakeyama K. 5-Fluorouracil cardiotoxicity induced by alpha-fluoro-beta-alanine. Int J Clin Oncol. 2005;10(6):441-443. doi:10.1007/s10147-005-0516-7

24. de Forni M, Malet-Martino MC, Jaillais P, et al. Cardiotoxicity of high-dose continuous infusion fluorouracil: a prospective clinical study. $J$ Clin Oncol. 1992;10(11):1795-1801. doi:10.1200/JCO.1992.10.11.1795

25. Upshaw JN, O'Neill A, Carver JR, et al. Fluoropyrimidine cardiotoxicity: time for a contemporaneous appraisal. Clin Colorectal Cancer. 2019;18(1):44-51. doi:10.1016/j.clcc.2018.08.001

26. Holubec L, Topolcan O, Finek J, et al. Dynamic monitoring of cardio-specific markers and markers of thyroid gland function in cancer patientsa pilot study. Anticancer Res. 2007;27(4A):1883-1886.

27. Salepci T, Seker M, Uyarel H, et al. 5-Fluorouracil induces arterial vasoconstrictions but does not increase angiotensin II levels. Med Oncol. 2010;27(2):416-420. doi:10.1007/s12032-009-9226-8

28. Abdel-Rahman O. 5-fluorouracil-related cardiotoxicity; findings from five randomized studies of 5-fluorouracil-based regimens in metastatic colorectal cancer. Clin Colorectal Cancer. 2019;18(1):58-63. doi:10.1016/j.clcc.2018.10.006

29. Kwakman JJM, Simkens LHJ, Mol L, Kok WEM, Koopman M, Punt CJA. Incidence of capecitabine-related cardiotoxicity in different treatment schedules of metastatic colorectal cancer: a retrospective analysis of the CAIRO studies of the Dutch Colorectal Cancer Group. Eur $J$ Cancer. 2017;76:93-99.

30. Balloni L, Porta C, Rossi S, et al. Left ventricular function in colon cancer patients receiving adjuvant fluoro-folate chemotherapy: an echocardiographic study. Oncol Rep. 2000;7(4):887-890. doi:10.3892/or.7.4.887

31. Khan MA, Masood N, Husain N, Ahmad B, Aziz T, Naeem A. A retrospective study of cardiotoxicities induced by 5-fluouracil (5-FU) and 5-FU based chemotherapy regimens in Pakistani adult cancer patients at Shaukat Khanum Memorial Cancer Hospital \& Research Center. $J$ Pak Med Assoc. 2012;62(5):430-434.

32. Akhtar SS, Salim KP, Bano ZA. Symptomatic cardiotoxicity with high-dose 5-fluorouracil infusion: a prospective study. Oncology. 1993;50 (6):441-444. doi:10.1159/000227226

33. Kosmas C, Kallistratos MS, Kopterides P, et al. Cardiotoxicity of fluoropyrimidines in different schedules of administration: a prospective study. J Cancer Res Clin Oncol. 2008;134(1):75-82. doi:10.1007/s00432-007-0250-9

34. Meyer CC, Calis KA, Burke LB, Walawander CA, Grasela TH. Symptomatic cardiotoxicity associated with 5-fluorouracil. Pharmacotherapy. 1997;17(4):729-736.

35. Tsavaris N, Kosmas C, Vadiaka M, et al. 5-fluorouracil cardiotoxicity is a rare, dose and schedule-dependent adverse event: a prospective study. $J$ BUON. 2005;10(2):205-211.

36. Van Cutsem E, Hoff PM, Blum JL, Abt M, Osterwalder B. Incidence of cardiotoxicity with the oral fluoropyrimidine capecitabine is typical of that reported with 5-fluorouracil. Ann Oncol. 2002;13(3):484-485. doi:10.1093/annonc/mdf108

37. Labianca R, Beretta G, Clerici M, Fraschini P, Luporini G. Cardiac toxicity of 5-fluorouracil: a study on 1083 patients. Tumori J. 1982;68 (6):505-510. doi:10.1177/030089168206800609

38. Meydan N, Kundak I, Yavuzsen T, et al. Cardiotoxicity of de Gramont's Regimen: incidence, clinical characteristics and long-term follow-up. Jpn J Clin Oncol. 2005;35(5):265-270. doi:10.1093/jjco/hyi071

39. Jensen SA, Sørensen JB. Risk factors and prevention of cardiotoxicity induced by 5-fluorouracil or capecitabine. Cancer Chemother Pharmacol. 2006;58(4):487-493. doi:10.1007/s00280-005-0178-1

40. Zafar A, Drobni ZD, Mosarla R, et al. The incidence, risk factors, and outcomes with 5-fluorouracil-associated coronary vasospasm. JACC CardioOncol. 2021;3(1):101-109. doi:10.1016/j.jaccao.2020.12.005

41. Anand AJ. Fluorouracil cardiotoxicity. Ann Pharmacother. 1994;28(3):374-378. doi:10.1177/106002809402800314

42. Gronich N, Lavi I, Barnett-Griness O, Saliba W, Abernethy DR, Rennert G. Tyrosine kinase-targeting drugs-associated heart failure. $\mathrm{Br}$ $J$ Cancer. 2017;116(10):1366-1373. doi:10.1038/bjc.2017.88

43. Cheng H, Gammon D, Dutton TM, Piperdi B. Panitumumab-related hypomagnesemia in patients with colorectal cancer. Hosp Pharm. 2009;44 (3):234-238. doi:10.1310/hpj4403-234

44. Abdel-Qadir H, Ethier J-L, Lee DS, Thavendiranathan P, Amir E. Cardiovascular toxicity of angiogenesis inhibitors in treatment of malignancy: a systematic review and meta-analysis. Cancer Treat Rev. 2017;53:120-127. doi:10.1016/j.ctrv.2016.12.002

45. Scappaticci FA, Skillings JR, Holden SN, et al. Arterial thromboembolic events in patients with metastatic carcinoma treated with chemotherapy and bevacizumab. J Natl Cancer Inst. 2007;99(16):1232-1239. doi:10.1093/jnci/djm086

46. Milano G, Etienne MC, Pierrefite V, Barberi-Heyob M, Deporte-Fety R, Renée N. Dihydropyrimidine dehydrogenase deficiency and fluorouracil-related toxicity. Br J Cancer. 1999;79(3-4):627-630. doi:10.1038/sj.bjc.6690098

47. Virani SA, Dent S, Brezden-Masley C, et al. Canadian Cardiovascular Society guidelines for evaluation and management of cardiovascular complications of cancer therapy. Can J Cardiol. 2016;32(7):831-841. doi:10.1016/j.cjca.2016.02.078

48. Koca D, Salman T, Unek IT, et al. Clinical and electrocardiography changes in patients treated with capecitabine. Chemotherapy. 2011;57 (5):381-387. doi:10.1159/000331645

49. Robben NC, Pippas AW, Moore JO. The syndrome of 5-fluorouracil cardiotoxicity. An elusive cardiopathy. Cancer. 1993;71(2):493-509. doi:10.1002/1097-0142(19930115)71:2<493::AID-CNCR2820710235>3.0.CO;2-C

50. Ambrosy AP, Kunz PL, Fisher GA, Witteles RM. Capecitabine-induced chest pain relieved by diltiazem. Am J Cardiol. 2012;110 (11):1623-1626. doi:10.1016/j.amjcard.2012.07.026 
51. Eskilsson J, Albertsson M. Failure of preventing 5-fluorouracil cardiotoxicity by prophylactic treatment with verapamil. Acta Oncol. 1990;29 (8):1001-1003. doi:10.3109/02841869009091790

52. Akpek G, Hartshorn KL. Failure of oral nitrate and calcium channel blocker therapy to prevent 5-fluorouracil-related myocardial ischemia: a case report. Cancer Chemother Pharmacol. 1999;43(2):157-161. doi:10.1007/s002800050877

53. Cianci G, Morelli MF, Cannita K, et al. Prophylactic options in patients with 5-fluorouracil-associated cardiotoxicity. Br J Cancer. 2003;88 (10):1507-1509. doi:10.1038/sj.bjc.6600967

54. Clasen SC, Ky B, O'Quinn R, Giantonio B, Teitelbaum U, Carver JR. Fluoropyrimidine-induced cardiac toxicity: challenging the current paradigm. J Gastrointest Oncol. 2017;8(6):970-979. doi:10.21037/jgo.2017.09.07

55. Bathina JD, Yusuf SW. 5-fluorouracil-induced coronary vasospasm. J Cardiovasc Med. 2010;11(4):281-284. doi:10.2459/ JCM.0b013e32832e934b

56. Saneeymehri SS, Markey KR, Mahipal A. Paradoxical effect of capecitabine in 5-fluorouracil-induced cardiotoxicity: a case vignette and literature review. J Oncol Pharm Pract. 2016;22(3):552-555. doi:10.1177/1078155215579303

57. Guo X-D, Harold N, Saif MW, et al. Pharmacokinetic and pharmacodynamic effects of oral eniluracil, fluorouracil and leucovorin given on a weekly schedule. Cancer Chemother Pharmacol. 2003;52(1):79-85. doi:10.1007/s00280-003-0613-0

58. Kikuchi K, Majima S, Murakami M. [Clinical survey on cardiotoxicity of tegafur (FT-207)-compilation of a nationwide survey]. Gan To Kagaku Ryoho. 1982;9(8):1482-1488. Japanese.

59. Marsh JC, Catalano P, Huang J, et al. Eastern Cooperative Oncology Group Phase II trial (E4296) of oral 5-fluorouracil and eniluracil as a 28-day regimen in metastatic colorectal cancer. Clin Colorectal Cancer. 2002;2(1):43-50. doi:10.3816/CCC.2002.n.010

60. Yip D, Karapetis C, Strickland AH, et al. A dose-escalating study of oral eniluracil/5-fluorouracil plus oxaliplatin in patients with advanced gastrointestinal malignancies. Ann Oncol. 2003;14(6):864-866. doi:10.1093/annonc/mdg254

61. Yamada Y, Hamaguchi T, Goto M, et al. Plasma concentrations of 5-fluorouracil and F-beta-alanine following oral administration of S-1, a dihydropyrimidine dehydrogenase inhibitory fluoropyrimidine, as compared with protracted venous infusion of 5-fluorouracil. $\mathrm{Br} J \mathrm{Cancer}$. 2003;89(5):816-820. doi:10.1038/sj.bjc.6601224

62. Boku N, Yamamoto S, Fukuda H, et al. Fluorouracil versus combination of irinotecan plus cisplatin versus S-1 in metastatic gastric cancer: a randomised phase 3 study. Lancet Oncol. 2009;10(11):1063-1069. doi:10.1016/S1470-2045(09)70259-1

63. Nagashima F, Ohtsu A, Yoshida S, Ito K. Japanese nationwide post-marketing survey of S-1 in patients with advanced gastric cancer. Gastric Cancer. 2005;8(1):6-11. doi:10.1007/s10120-004-0306-3

64. Vaflard P, Ederhy S, Torregrosa C, André T, Cohen R, Lopez-Trabada D. [Fluoropyrimidines cardiac toxicity: 5-fluorouracil, capecitabine, compound S-1 and trifluridine/tipiracil]. Bull Cancer. 2018;105(7-8):707-719. French. doi:10.1016/j.bulcan.2018.05.005

65. Mayer RJ, Van Cutsem E, Falcone A, et al. Randomized trial of TAS-102 for refractory metastatic colorectal cancer. $N$ Engl J Med. 2015;372 (20):1909-1919. doi:10.1056/NEJMoa1414325

66. Lopez CA, Azimi-Nekoo E, Chung SY, Newman J, Shen J, Saif WM. Meta-analysis and systematic review of the cardiotoxicity of TAS-102. J Clin Oncol. 2020;38(15_suppl):e16053-e16053. doi:10.1200/JCO.2020.38.15_suppl.e16053

67. Kelly C, Bhuva N, Harrison M, Buckley A, Saunders M. Use of raltitrexed as an alternative to 5-fluorouracil and capecitabine in cancer patients with cardiac history. Eur J Cancer. 2013;49(10):2303-2310. doi:10.1016/j.ejca.2013.03.004

68. Ransom D, Wilson K, Fournier M, et al. Final results of Australasian Gastrointestinal Trials Group ARCTIC study: an audit of raltitrexed for patients with cardiac toxicity induced by fluoropyrimidines $\dagger$. Ann Oncol. 2014;25(1):117-121. doi:10.1093/annonc/mdt479

69. Deboever G, Hiltrop N, Cool M, Lambrecht G. Alternative treatment options in colorectal cancer patients with 5-fluorouracil- or capecitabineinduced cardiotoxicity. Clin Colorectal Cancer. 2013;12(1):8-14.

70. Lembersky BC, Wieand HS, Petrelli NJ, et al. Oral uracil and tegafur plus leucovorin compared with intravenous fluorouracil and leucovorin in stage II and III carcinoma of the colon: results from National Surgical Adjuvant Breast and Bowel Project Protocol C-06. J Clin Oncol. 2006;24 (13):2059-2064. doi:10.1200/JCO.2005.04.7498

71. Shimada Y, Hamaguchi T, Mizusawa J, et al. Randomised phase III trial of adjuvant chemotherapy with oral uracil and tegafur plus leucovorin versus intravenous fluorouracil and levofolinate in patients with stage III colorectal cancer who have undergone Japanese D2/D3 lymph node dissection: final results of JCOG0205. Eur J Cancer. 2014;50(13):2231-2240. doi:10.1016/j.ejca.2014.05.025

72. Yoshida M, Ishiguro M, Ikejiri K, et al. S-1 as adjuvant chemotherapy for stage III colon cancer: a randomized phase III study (ACTS-CC trial). Ann Oncol. 2014;25(9):1743-1749. doi:10.1093/annonc/mdu232

73. Hamaguchi T, Shimada Y, Mizusawa J, et al. Capecitabine versus S-1 as adjuvant chemotherapy for patients with stage III colorectal cancer (JCOG0910): an open-label, non-inferiority, randomised, phase 3, multicentre trial. Lancet Gastroenterol Hepatol. $2018 ; 3(1): 47-56$. doi:10.1016/S2468-1253(17)30297-2

74. Sharif S, O’Connell MJ, Yothers G, Lopa S, Wolmark N. FOLFOX and FLOX regimens for the adjuvant treatment of resected stage II and III colon cancer. Cancer Invest. 2008;26(9):956-963. doi:10.1080/07357900802132550

75. Chakrabarti S, Sara J, Lobo R, et al. Bolus 5-fluorouracil (5-FU) in combination with oxaliplatin is safe and well tolerated in patients who experienced coronary vasospasm with infusional 5-FU or capecitabine. Clin Colorectal Cancer. 2019;18(1):52-57. doi:10.1016/j. clcc.2018.09.006

76. Haller DG, Catalano PJ, Macdonald JS, et al. Phase III study of fluorouracil, leucovorin, and levamisole in high-risk stage II and III colon cancer: final report of Intergroup 0089. J Clin Oncol. 2005;23(34):8671-8678. doi:10.1200/JCO.2004.00.5686

77. Douillard J-Y, Hoff PM, Skillings JR, et al. Multicenter phase III study of uracil/tegafur and oral leucovorin versus fluorouracil and leucovorin in patients with previously untreated metastatic colorectal cancer. J Clin Oncol. 2002;20(17):3605-3616. doi:10.1200/JCO.2002.04.123

78. Bennouna J, Saunders M, Douillard J-Y. The role of UFT in metastatic colorectal cancer. Oncology. 2009;76(5):301-310. doi:10.1159/ 000209334

79. Ohtsu A, Baba H, Sakata Y, et al. Phase II study of S-1, a novel oral fluorophyrimidine derivative, in patients with metastatic colorectal carcinoma. S-1 Cooperative Colorectal Carcinoma Study Group. Br J Cancer. 2000;83(2):141-145. doi:10.1054/bjoc.2000.1236

80. Shirao K, Ohtsu A, Takada H, et al. Phase II study of oral S-1 for treatment of metastatic colorectal carcinoma. Cancer. 2004;100 (11):2355-2361. doi:10.1002/cncr.20277 
81. Park YS, Lim HY, Lee J, et al. A randomized phase III study of sox (S-1/oxaliplatin) versus COX (capecitabine/oxaliplatin) in patients with advanced colorectal cancer. J Clin Oncol. 2011;29(15_suppl):3524. doi:10.1200/jco.2011.29.15_suppl.3524

82. Muro K, Boku N, Shimada Y, et al. Irinotecan plus S-1 (IRIS) versus fluorouracil and folinic acid plus irinotecan (FOLFIRI) as second-line chemotherapy for metastatic colorectal cancer: a randomised Phase 2/3 non-inferiority study (FIRIS study). Lancet Oncol. 2010;11(9):853-860. doi:10.1016/S1470-2045(10)70181-9

83. Popov I, Carrato A, Sobrero A, et al. Raltitrexed (Tomudex) versus standard leucovorin-modulated bolus 5-fluorouracil: results from the randomised phase III Pan-European Trial in Adjuvant Colon Cancer 01 (PETACC-1). Eur J Cancer. 2008;44(15):2204-2211. doi:10.1016/j. ejca.2008.07.002

84. Cocconi G, Cunningham D, Van Cutsem E, et al. Open, randomized, multicenter trial of raltitrexed versus fluorouracil plus high-dose leucovorin in patients with advanced colorectal cancer. Tomudex Colorectal Cancer Study Group. J Clin Oncol. 1998;16(9):2943-2952. doi:10.1200/JCO.1998.16.9.2943

85. Cunningham D, Zalcberg JR, Rath U, et al. Final results of a randomised trial comparing "Tomudex" (raltitrexed) with 5-fluorouracil plus leucovorin in advanced colorectal cancer. 'Tomudex' Colorectal Cancer Study Group. Ann Oncol. 1996;7(9):961-965. doi:10.1093/oxfordjournals.annonc.a010800

86. Maughan TS, James RD, Kerr DJ, et al. Comparison of survival, palliation, and quality of life with three chemotherapy regimens in metastatic colorectal cancer: a multicentre randomised trial. Lancet. 2002;359(9317):1555-1563. doi:10.1016/S0140-6736(02)08514-8

87. Grávalos C, García-Girón C, Salut A, et al. TOMOX compared to FOLFOX4 as first-line treatment in patients (pts) with advanced colorectal cancer (ACRC): results of a multicenter randomized phase II trial. $J$ Clin Oncol. 2005;23(16_suppl):3563. doi:10.1200/ jco.2005.23.16_suppl.3563

88. Laudani A, Gebbia V, Leonardi V, et al. Activity and toxicity of oxaliplatin plus raltitrexed in 5-fluorouracil refractory metastatic colorectal adeno-carcinoma. Anticancer Res. 2004;24(2C):1139-1142.

89. Scheithauer W, Kornek GV, Schuell B, et al. Second-line treatment with oxaliplatin + raltitrexed in patients with advanced colorectal cancer failing fluoropyrimidine/leucovorin-based chemotherapy. Ann Oncol. 2001;12(5):709-714. doi:10.1023/A:1011194712661

90. Wilson KS, Fitzgerald CA, Barnett JB, Gill S, Khoo KE. Adjuvant therapy with raltitrexed in patients with colorectal cancer intolerant of 5-fluorouracil: British Columbia Cancer Agency experience. Cancer Invest. 2007;25(8):711-714. doi:10.1080/07357900701518388

91. Feliu J, Castañón C, Salud A, et al. Phase II randomised trial of raltitrexed-oxaliplatin vs raltitrexed-irinotecan as first-line treatment in advanced colorectal cancer. Br J Cancer. 2005;93(11):1230-1235. doi:10.1038/sj.bjc.6602860

92. Carnaghi C, Rimassa L, Garassino I, et al. Irinotecan and raltitrexed: an active combination in advanced colorectal cancer. Ann Oncol. 2002;13 (9):1424-1429. doi:10.1093/annonc/mdf229

93. Feliu J, Salud A, Escudero P, et al. Irinotecan plus raltitrexed as first-line treatment in advanced colorectal cancer: a phase II study. Br J Cancer. 2004;90(8):1502-1507. doi:10.1038/sj.bjc.6601713

94. Aparicio J, Vicent JM, Maestu I, et al. Multicenter phase II trial evaluating a three-weekly schedule of irinotecan plus raltitrexed in patients with 5-fluorouracil-refractory advanced colorectal cancer. Ann Oncol. 2003;14(7):1121-1125. doi:10.1093/annonc/mdg285

95. Cutsem EV, Danielewicz I, Saunders MP, et al. Trifluridine/tipiracil plus bevacizumab in patients with untreated metastatic colorectal cancer ineligible for intensive therapy: the randomized TASCO1 study. Ann Oncol. 2020;31(9):1160-1168. doi:10.1016/j.annonc.2020.05.024

96. Van Cutsem E, Danielewicz I, Saunders MP, et al. Phase II study evaluating trifluridine/tipiracil + bevacizumab and capecitabine + bevacizumab in first-line unresectable metastatic colorectal cancer (mCRC) patients who are noneligible for intensive therapy (TASCO1): results of the final analysis on the overall survival. $J$ Clin Oncol. 2021;39(3_suppl):14.

97. André T, Saunders M, Kanehisa A, et al. First-line trifluridine/tipiracil plus bevacizumab for unresectable metastatic colorectal cancer: SOLSTICE study design. Future Oncol. 2020;16(4):21-29. doi:10.2217/fon-2019-0786

98. Yoshino T, Oki E, Nozawa H, et al. Rationale and design of the TRUSTY study: a randomised, multicentre, open-label phase II/III study of trifluridine/tipiracil plus bevacizumab versus irinotecan, fluoropyrimidine plus bevacizumab as second-line treatment in patients with metastatic colorectal cancer progressive during or following first-line oxaliplatin-based chemotherapy. ESMO Open. 2018;3(5):e000411. doi:10.1136/ esmoopen-2018-000411

99. Saltz LB, Cox JV, Blanke C, et al. Irinotecan plus fluorouracil and leucovorin for metastatic colorectal cancer. Irinotecan Study Group. $N$ Engl $J$ Med. 2000;343(13):905-914. doi:10.1056/NEJM200009283431302

100. Cunningham D, Pyrhönen S, James RD, et al. Randomised trial of irinotecan plus supportive care versus supportive care alone after fluorouracil failure for patients with metastatic colorectal cancer. Lancet. 1998;352(9138):1413-1418. doi:10.1016/S0140-6736(98)02309-5

101. Rougier P, Van Cutsem E, Bajetta E, et al. Randomised trial of irinotecan versus fluorouracil by continuous infusion after fluorouracil failure in patients with metastatic colorectal cancer. Lancet. 1998;352(9138):1407-1412. doi:10.1016/S0140-6736(98)03085-2

102. Cunningham D, Humblet Y, Siena S, et al. Cetuximab monotherapy and cetuximab plus irinotecan in irinotecan-refractory metastatic colorectal cancer. $N$ Engl J Med. 2004;351(4):337-345. doi:10.1056/NEJMoa033025

103. Sobrero AF, Maurel J, Fehrenbacher L, et al. EPIC: phase III trial of cetuximab plus irinotecan after fluoropyrimidine and oxaliplatin failure in patients with metastatic colorectal cancer. J Clin Oncol. 2008;26(14):2311-2319. doi:10.1200/JCO.2007.13.1193

104. Fischer von Weikersthal L, Schalhorn A, Stauch M, et al. Phase III trial of irinotecan plus infusional 5-fluorouracil/folinic acid versus irinotecan plus oxaliplatin as first-line treatment of advanced colorectal cancer. Eur J Cancer. 2011;47(2):206-214. doi:10.1016/j.ejca.2010.09.022

105. Haller DG, Rothenberg ML, Wong AO, et al. Oxaliplatin plus irinotecan compared with irinotecan alone as second-line treatment after single-agent fluoropyrimidine therapy for metastatic colorectal carcinoma. J Clin Oncol. 2008;26(28):4544-4550. doi:10.1200/ JCO.2008.17.1249

106. Curigliano G, Lenihan D, Fradley M, et al. Management of cardiac disease in cancer patients throughout oncological treatment: ESMO consensus recommendations. Ann Oncol. 2020;31(2):171-190. doi:10.1016/j.annonc.2019.10.023

107. Fradley MG, Beckie TM, Brown SA, et al. Recognition, prevention, and management of arrhythmias and autonomic disorders in cardio-oncology: a scientific statement from the American Heart Association. Circulation. 2021;144(3):e41-e55. doi:10.1161/ CIR.0000000000000986 
108. Zamorano JL, Lancellotti P, Rodriguez Muñoz D, et al. 2016 ESC Position Paper on cancer treatments and cardiovascular toxicity developed under the auspices of the ESC Committee for Practice Guidelines: the Task Force for cancer treatments and cardiovascular toxicity of the European Society of Cardiology (ESC). Eur Heart J. 2016;37(36):2768-2801. doi:10.1093/eurheartj/ehw211

109. Sara JD, Kaur J, Khodadadi R, et al. 5-fluorouracil and cardiotoxicity: a review. Ther Adv Med Oncol. 2018;10:1758835918780140. doi:10.1177/1758835918780140

110. Bamat MK, Tremmel R, O’Neil JD, von Borstel R. Uridine triacetate: an orally administered, life-saving antidote for 5-FU overdose. $J$ Clin Oncol. 2010;28(15_suppl):9084. doi:10.1200/jco.2010.28.15_suppl.9084

111. Ma WW, Saif MW, El-Rayes BF, et al. Emergency use of uridine triacetate for the prevention and treatment of life-threatening 5-fluorouracil and capecitabine toxicity. Cancer. 2017;123(2):345-356. doi:10.1002/cncr.30321

112. Raber I, Frazer MB, Zerillo JA, Asnani A. Uridine triacetate for severe fluoropyrimidine cardiotoxicity in a patient with thymidylate synthase gene variants. JACC CardioOncol. 2020;2(2):329-332. doi:10.1016/j.jaccao.2020.04.005

113. Ison G, Beaver JA, McGuinn WD, et al. FDA approval: uridine triacetate for the treatment of patients following fluorouracil or capecitabine overdose or exhibiting early-onset severe toxicities following administration of these drugs. Clin Cancer Res. 2016;22(18):4545-4549. doi:10.1158/1078-0432.CCR-16-0638

114. Carmichael J, Popiela T, Radstone D, et al. Randomized comparative study of tegafur/uracil and oral leucovorin versus parenteral fluorouracil and leucovorin in patients with previously untreated metastatic colorectal cancer. J Clin Oncol. 2002;20(17):3617-3627. doi:10.1200/ JCO.2002.10.129

\section{Publish your work in this journal}

Cancer Management and Research is an international, peer-reviewed open access journal focusing on cancer research and the optimal use of preventative and integrated treatment interventions to achieve improved outcomes, enhanced survival and quality of life for the cancer patient. The manuscript management system is completely online and includes a very quick and fair peer-review system, which is all easy to use. Visit http://www.dovepress.com/testimonials.php to read real quotes from published authors.

Submit your manuscript here: https://www.dovepress.com/cancer-management-and-research-journal 